\title{
A Combination Technique for Accurate Location and Debridement of Pathological Lesion in Femoral Head
}

\author{
Qilin $\mathrm{Lu}^{1}$, Jin Tang ${ }^{1}$, Xianhua Cai ${ }^{2}$, Shijun $\mathrm{Wei}^{2}$ and Xugui $\mathrm{Li}^{1}$ \\ ${ }^{1}$ Department of Orthopaedics, Hubei 672 Orthopaedics Hospital of Integrated Chinese and Western Medicine, Wuhan, China \\ ${ }^{2}$ Department of Orthopaedics, The General Hospital of Central Theater Command, Wuhan, China
}

\begin{abstract}
Debridement with bone grafting is a feasible and effective joint preserving method for early stage avascular necrosis and benign tumor of the femoral head before collapse. Although common CT and X-ray guide techniques were helpful, high dose of intraoperative radiation, inaccurate intraoperative location of pathological region, unverifiable of debridement, and longer operative duration still challenge the surgeons during the operation. With the development of new medical technology and instrument, a combination technique employing 3-D navigation endoscopy could overcome above mentioned drawbacks during the operation; and can also provide satisfactory clinical effect. This method has not been described in previous literature.
\end{abstract}

Key Words: 3-D navigation, Endoscopy, Femoral head, Debridement.

How to cite this article: Lu Q, Tang J, Cai X, Wei S, Li X. A Combination Technique for Accurate Location and Debridement of Pathological Lesion in Femoral Head. J Coll Physicians Surg Pak 2020; 30(12):1335-1338.

\section{INTRODUCTION}

Avascular necrosis and benign tumor of the femoral head are common pathological lesions. Both can cause collapse or fracture of femoral head in the later period. ${ }^{1,2}$ Therefore, scientific intervention in early stage is essential for the morphology of femoral head and function of hip joint. It has been established that debridement with bone grafting is a feasible and effective joint preserving method for pathological lesion of femoral head before collapse. ${ }^{3}$ Over the past decades, this technique has been developing for various techniques. ${ }^{4}$ However, these still have 4 obvious drawbacks including inaccurate intraoperative aim, high dose of intraoperative radiation, unverifiable thoroughness of debridement and longer operative duration even if with the assistance of $X$-rays and $\mathrm{CT}^{5}{ }^{5}$

The authors describe a technique, combined 3-D navigation and endoscope (hip arthroscope), in order to make up for drawbacks of traditional techniques in debridement with bone grafting for treating pathological lesion before the femoral head collapse. To the best of the authors' knowledge, this combination technique or concepthas not been reported in previous literature.

Correspondence to: Dr. Xugui Li, Department of Orthopaedics, Hubei 672 Orthopaedics Hospital of Integrated Chinese $\square$ Western Medicine, No. 279, Luoyu Road, Hongshan District, Wuhan, Hubei Province, China

E-mail: bjxarlene@aliyun.com

Received: December 09, 2019; Revised: February 12, 2020;

Accepted: March 09, 2020

DOI: https://doi.org/10.29271/jcpsp.2020.12.1335

\section{Technique:}

The patient was placed in supine position. The buttock of the lesion side was slightly elevated with a pillow. Medical binding belts were applied to keep the pathological hip joint stable. The surgical region was disinfected and prepared. 3-D navigation system (Stryker, USA) was registered and activated. Subsequently, a scanning for this hip joint was performed, and the data was instantly transferred to navigation system screen, which showed the cross-section, coronal, sagittal view and 3-D construction of hip (Figure 1a). Incision with $3 \mathrm{~cm}$ was longitudinally made along the lateral side of thigh. Lateral bone cortex at the level of minor trochanter was exposed and regarded as entry point. Then the induced navigated cannulated sleeve of navigation system was used. Once this sleeve touched the entry point of cortical bone, green virtual guide arrows on the screen were immediately created in the femurneck and head by cross-section, coronal, sagittal view (Figure 1a). Adjustment in the angle of induced navigated sleeve outside can simulate routes in the bone to predict the optimal path, which was revealing the exact distance and direction to lesion region. After the precise location, a $2.0 \mathrm{~mm}$ guide pin was inserted through the cannulated sleeve to follow the predicted optimal path.

Cannulated drill with $12 \mathrm{~mm}$ in diameter directly advanced along guide pin to reach the lesion region, according to distance, which was predicted from previous optimal path (Figure 1b). After the drill was withdrawn, hip arthroscope was inserted into bony track as endoscope to check the inside wall of pathological lesion region and washed out the bone debris. Under the clear view, the surgeon was able to notice the inside of lesion region and perform the debridement by different radians curettes (Figure 1c). Alternate application of arthros- 
cope and curette was performed until the pathological part was thoroughgoing scraped off. Once the condition that healthy bone began to bleed from the original pathological color was observed under the arthroscope, the debridement was regarded as finish (Figure $1 \mathrm{~d}, \mathrm{e}$ ).

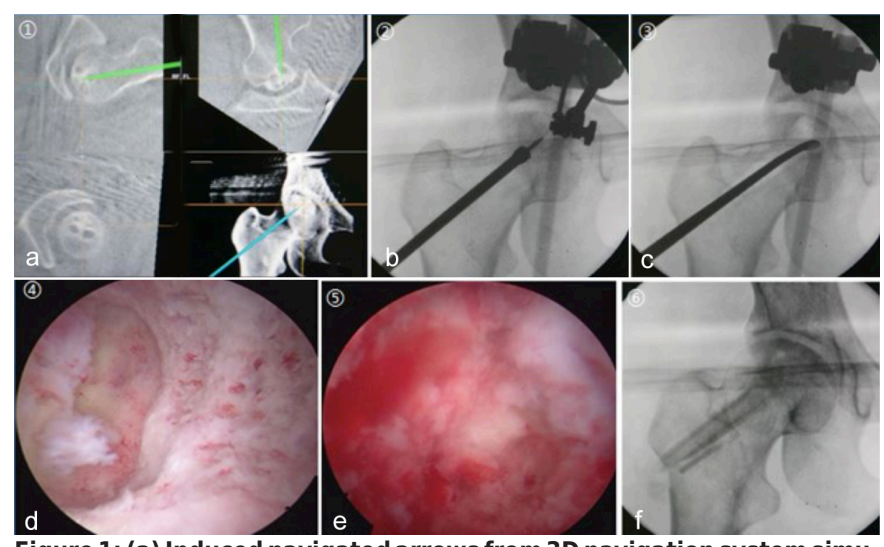

Figure 1: (a) Induced navigated arrows from 3D navigation system simulates the routes in the bone to obtain the optimal path to the lesion region. (b) guide pin with cannulated drill. (c) Debridement with curette. (d, e) Before and after debridement condition from endoscope. (f) Postoperative view with bone graft materials.
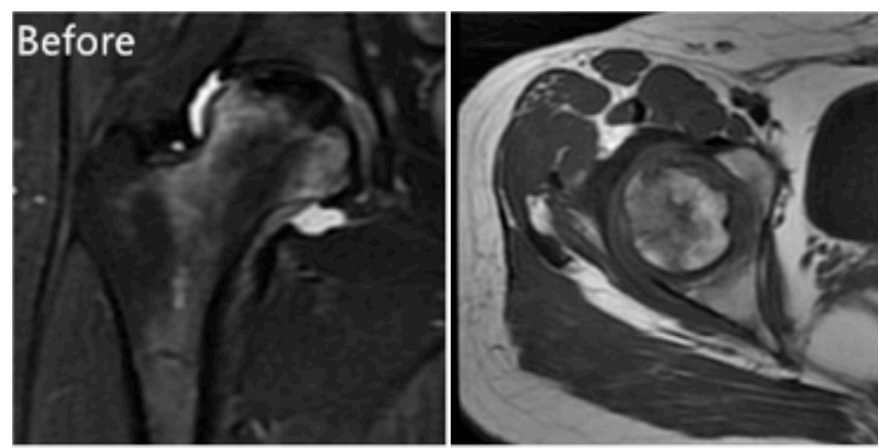

\section{After}

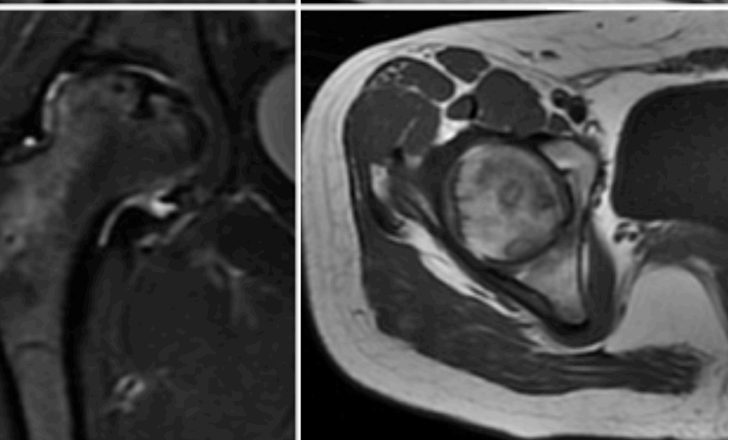

Figure 2: MRI showed the patient A's condition of pathological femoral head before and 18 months after surgery.

The autologous iliac bone was harvested and cut into small pieces with $5 \mathrm{~mm}$ in cubic. Those bones were implanted into the cavity after the debridement. An allogeneic fibula with pillar shape was implanted into the bony track, not only to compact inner new planted bone but also to support the femoral neck and cartilage (Figure 1f).

\section{Cases and results:}

One patient with glucocorticoid-induced osteonecrosis (case Figure 2) and the other one with aneurysmal bone cyst (case B) in the femoral head were treated by this technique before their femoral head collapse. Postoperatively, they were allowed partial weight bearing with crutches for six weeks and recovered to full bearing in three months. MRI and Harris score were used to evaluate their recovery conditions at follow-up period. Harris score of case A escalated from 62 to 90 , and MRI T2WI images showed that the femoral head bone graft survived well, the original necrosis area was significantly reduced, and intra-articular inflammation was effectively controlled at the $18^{\text {th }}$ month after surgery (Figure 2). Harris score of case B elevated from 72 to 93 , and MRI T2WI images showed the morphology of the femoral head was recovered satisfactorily with well survival of grafted bone at the $6^{\text {th }}$ month after surgery (Figure 3).

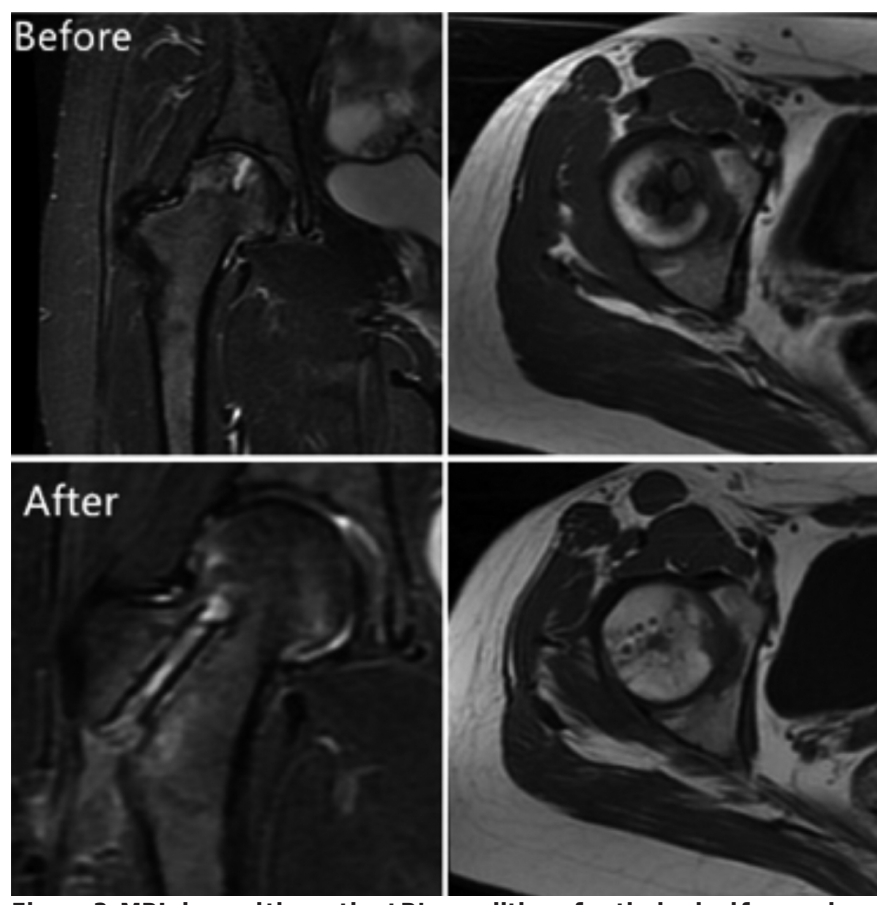

Figure 3: MRI showed the patient B's condition of pathological femoral head before and 6 months after surgery.

\section{DISCUSSION}

Avascular necrosis of the femoral head (ANFH) refers to the necrosis and collapse of femoral head due to its blood supply disorder, secondary to harmful factors such as glucocorticoid, alcohol, trauma. ${ }^{6}$ Aneurysmal bone cysts is a kind of benign lesions, which is usually encountered in children and adolescents. The main concern about it, is the pathological fracture. ${ }^{2}$ Debridement with bone grafting may remove the necrotic bone, reduce the internal pressure to improve the internal blood supply, and induce new bone development before the collapse of femoral head. Over the past decades, although this technique has developed and diversified into different methods and with assistance of common CT or X-ray, there are still some drawbacks, which need to be resolved. With the invention and application of new medical equipment, it highlights the advantages of combination of 3-D navigation and endoscopy. 3-D navigation guidance can be used for precise target location in femoral head, and endoscope may assist the evaluation of lesion tissues debridement. Finally, bone grafting is performed though the bony track. This combination technique may make up the shortcomings of previous methods. 
Owing to the multiplanar imaging function, real-time computer tomography (CT) technique has been used for osteonecrosis of the femoral head. ${ }^{7}$ 3-D navigation system can enable surgeons to visually simulate routes without real drilling. Therefore, 3-D navigation system has more advantages over real-time CT in minimal invasive and accurate guidance. 3-D navigation in this study can precisely perform target location. Compared with fluoroscopy, 3-D navigation provides actual hip with cross-section, coronal and sagittal view only by oncescanning, which facilitates surgeons to control direction and depth of guide pin; and dramatically reduce the intraoperative radiation. By adjusting the direction of induced navigated drill sleeve allows green arrow to individually provide optimal path, which guides pin cross the main axis direction of pathological region. Arthroscope, by following this straight direction, may easily perform the debridement at nextstep.

The application of arthroscope as an endoscope in percutaneous debridement for osteonecrosis of the femoral head was introduced by Pierannunzii. ${ }^{5}$ The water from this equipment can wash out bone debris, and the camera in equipment provide clear view inside. Visualisation was the greatest contribution from arthroscope to the removal of pathological region. With the visualising view, thorough debridement could be well performed with much lower risk of iatrogenic bone injury. Healthy bone, preserved as much as possible, could improve the survival rate of bone graft by providing good blood supply bed.

The high survival rate of bone graft has significant contribution to the long-term function of femoral head. ${ }^{8}$ 3D-guided precise location could reduce the injury risk of vascular supplies for femoral head. Visualised debridement with the assistance of arthroscope could provide three-dimensional good blood supply bed for bone graft. In addition, enough compact on bone graft could enhance the contact area with bony bed. The autologous iliac bone might be unnecessary, if the necrotic region was not big enough. A cylindrical bone can be harvested by reamer along the guide pin from lateral of intertrochanter, which was high quality autologous bone for femoral head after lesion debridement. This operation can avoid complications from iliac donated region.

Many notices in present procedure need to be mentioned. For ANFH, indication for this technique should be strictly limited under the early-stage before the collapse of femoral head. Crescent sign in ANFH, identified as the critical value of the early-stage, reveals subchondral fracture of femoral head. For tumor, the size and location in the femoral head are vital factors for this technique. The internal fixation is recommended after these present procedures, if the tumor, even if without collapse, occupies large capacity or it is located at the border between femoral head and neck. During the navigation procedure, patients are required to keep still position. Otherwise, the accuracy will be affected. latrogenic cartilage injury lesion should be avoided even if the depth is limited for cannulate drill. Position of the guide pin needs to be closely observed when the cannulated drill advances it in case that it moves together ahead with drill to perforate the cartilage. Patients of old age, unstable hip joint, serious necrosis on the heavy-bearing zone or collapse, should be excluded.

\section{CONCLUSION}

3-Dnavigation technique combined with endoscope for percutaneous debridement with bone grafting has reasonable advantages in early staged pathological lesion of femoral head. Clinically, it has been verified that this technique has the following advantages over conventional ways: precise location, satisfied debridement with less intraoperative radiation, which could save more operative duration.

Those two cases have achieved the satisfied results from this technique. However, the long-term effect of those pathological lesionsstill need a further and largesamples study. The 3-D navigation and arthroscopic assistance are harmless to patients, and affordable from economic view. Meanwhile, both of them are mature clinical techniques. Therefore, this combined technique possesses potential application prospects in bone puncture guidance for diagnosis and treatment of malignant tumors, tuberculosis, and other benign tumor of femoral head.

\section{ETHICALAPPROVAL:}

This technique was approved by the Research Ethics Committee of 672 Hospital (No: 672HREC2016071) before the initiation of the research work.

\section{PATIENTS' CONSENT:}

The authors certify that they have obtained all appropriate patients' consents to publish the data concerning this case. The patients understand that their names and initials will not be published and efforts will be made to conceal their identity.

\section{CONFLICT OF INTEREST:}

The authors declared no conflict of interest.

\section{AUTHORS' CONTRIBUTION:}

QL: Surgical design and manuscript writing.

JT: Surgical operation.

XC, SW: Surgical design guidance and pictures collection.

XL: Surgical operation and manuscript proofreading.

\section{REFERENCES}

1. Cui L, Zhuang Q, Lin J, Jin J, Zhang K, Cao L, et al. Multicentric epidemiologic study on six thousand three hundred and ninety five cases of femoral head osteonecrosis in China. Int orthopaedics 2016; 40(2): 267-76.

2. Mascard E, Gomez-Brouchet A, Lambot K. Bone cysts: Unicameral and aneurysmal bone cyst. Orthop Traumatol Surg Res 2015; 101(1):119-27.

3. Zhang QY, Li ZR, Gao FQ. Pericollapse stage of osteonecrosis of the femoral head: A last chance for joint preservation. Chinese Med J 2018; 131(21):2589-98.

4. Wang J, Wang J, Zhang K, Wang F, Bao X. Bayesian network meta-analysis of the effectiveness of various interventions for nontraumatic osteonecrosis of the femoral head. 
Biomed Res Int 2018; 2018:2790163.

5. Pierannunzii L. Endoscopic and arthroscopic assistance in femoral head core decompression. Arthroscopy Tech 2012; 1(2):e225-30.

6. Fukushima W, Fujioka M, Kubo T, Tamakoshi A, Nagai M, Hirota Y. Nationwide epidemiologic survey of idiopathic osteonecrosis of the femoral head. Clin Orthopaed Res 2010; 468(10):2715-24.
7. Hu R, Lei P, Li B, Liu H, Yang X, Wen T, et al. Real-time computerised tomography assisted porous tantalum implant in ARCO stage I-II non-traumatic osteonecrosis of the femoral head: Minimum five-year follow up. Int Orthopaed 2018; 42(7):1535-44.

8. Yu PA, Peng KT, Huang TW, Hsu R. Injectable synthetic bone graft substitute combined with core decompression in the treatment of advanced osteonecrosis of the femoral head: A 5-year follow-up. Biomed J 2015; 38(3):257-61. 